\title{
DÜBLIN
}

Technological University Dublin

ARROW@TU Dublin

Articles

School of Manufacturing and Design

Engineering

2017

\section{A Simple Optical Fiber Interferometer Based Breathing Sensor}

Xixi Li

Tsinghua University

Dejun Liu

Technological University Dublin, dejun.liu@tudublin.ie

Rahul Kumar

Northumbria University

See next page for additional authors

Follow this and additional works at: https://arrow.tudublin.ie/engschmanart

\section{Recommended Citation}

Li, X., Liu, D. \& Kumar, R. (2017). A simple optical fiber interferometer based breathing sensor. Measurement Science and Technology, vol. 28, no. 3. doi: 10.1088/1361-6501/aa577d.

This Article is brought to you for free and open access by the School of Manufacturing and Design Engineering at ARROW@TU Dublin. It has been accepted for inclusion in Articles by an authorized administrator of ARROW@TU Dublin. For more information, please contact arrow.admin@tudublin.ie, aisling.coyne@tudublin.ie, gerard.connolly@tudublin.ie.

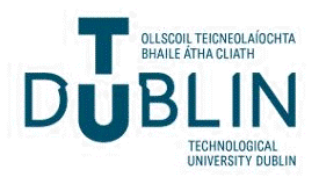




\section{Authors}

Xixi Li, Dejun Liu, Rahul Kumar, Wai Pang Ng, Yong-qing Fu, Jinhui Yuan, Chongxiu Yu, Yufeng Wu, Guorui Zhou, Gerald Farrell, Yuliya Semenova, and Qiang Wu 


\title{
A simple optical fiber interferometer based breathing sensor
}

\author{
Xixi Li ${ }^{1,6}$, Dejun Liu' ${ }^{2,6}$, Rahul Kumar' ${ }^{1,6}$, Wai Pang Ng${ }^{1}$, Yong-qing Fu ${ }^{1}$, \\ Jinhui Yuan ${ }^{3}$, Chongxiu $\mathbf{Y u}^{3}$, Yufeng Wu ${ }^{4}$, Guorui Zhou ${ }^{5}$, Gerald Farrell ${ }^{2}$, \\ Yuliya Semenova ${ }^{2}$ and Qiang $\mathbf{W u}^{1}$ \\ 1 Department of Mathematic, Physics and Electrical Engineering, Northumbria University, Newcastle
Upon Tyne, NE1 8ST, United Kingdom
2 Photonics Research Centre, Dublin Institute of Technology, Kevin Street, Dublin 8, Ireland
${ }^{3}$ State Key Laboratory of Information Photonics and Optical Communications, Beijing University of
Posts and Telecommunications, Beijing 100876, People's Republic of China
${ }^{4}$ Institute of Engineering, Lishui University, Zhejiang, People's Republic of China
5 Laser Fusion Research Center, China Academy of Engineering Physics, Mianyang 621900, People's
Republic of China \\ E-mail: qiang.wu@ northumbria.ac.uk and yuanjinhui81@163.com
}

Received 30 September 2016, revised 14 December 2016

Accepted for publication 6 January 2017

Published 2 February 2017

\begin{abstract}
A breathing sensor has been experimentally demonstrated based on a singlemode-multimodesinglemode (SMS) fiber structure which is attached to a thin plastic film in an oxygen mask. By detecting power variations due to the macro bending applied to the SMS section by each inhalation and exhalation process, the breath state can be monitored. The proposed sensor is capable of distinguishing different types of breathing conditions including regular and irregular breath state. The sensor can be used in a strong electric/magnetic field and radioactive testing systems such as magnetic resonance imaging (MRI) systems and computed tomography (CT) examinations where electrical sensors are restricted.
\end{abstract}

Keywords: optical fiber interferometer, optical sensor, breath sensor

(Some figures may appear in colour only in the online journal)

\section{Introduction}

Breathing rate is defined as the frequency of breaths taken within a certain amount of time. The typical breathing rate for a healthy adult at rest is between 12 and 20 breaths per minute. It is recognized as one of the most important human vital signs to assess physiological state. A lot of valuable information related to cardiac, neurological and pulmonary conditions can be obtained by analyzing the breathing data [1]. For example, patients' heart and lung diseases can be identified by monitoring breathing in a hospital setting.

There are various methods for breathing measurements such as monitoring the air exhaled through the nose and mouth, or external movement of the thoracic cavity/chest. Based on different working principles, a number of breath sensors have

${ }^{6}$ These authors have equal contributions to this paper. been proposed, such as sensors utilizing humidity or temperature measurements, capnometer and spirometer to monitor breathing behavior [2]. To date, most of the commercialized breath measurement devices are based on electronic sensors, which can be easily influenced by local electric/magnetic fields due to the presence of metallic parts or electrical conductive wires. Consequently, electronic breathing sensors are not suitable for applications associated with strong electric/ magnetic environments [3], for example, in a magnetic resonance imaging (MRI) system. However, in many practical situations it is important to monitor the respiratory rate and apnea during the use of MRI.

Fiber optic sensors have intrinsic advantages of fast response, very compact size, immunity to electromagnetic interference and remote sensing capabilities and hence have recently attracted growing attention in applications for breath monitoring. For example, Krehel et al [4] reported a 
(a)

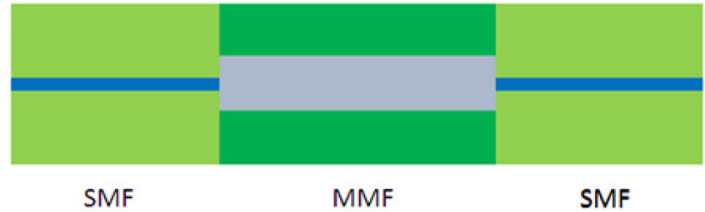

(b)

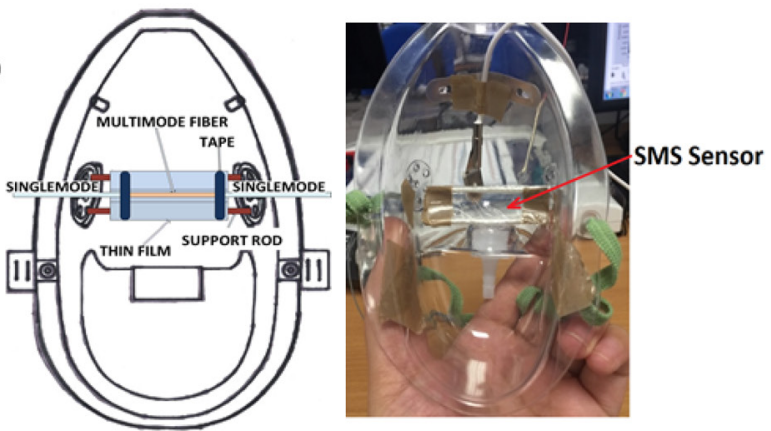

Figure 1. (a) Schematic diagram of an SMS fiber structure and (b) a schematic diagram and a photograph of the real prototype of the oxygen mask with embedded SMS.

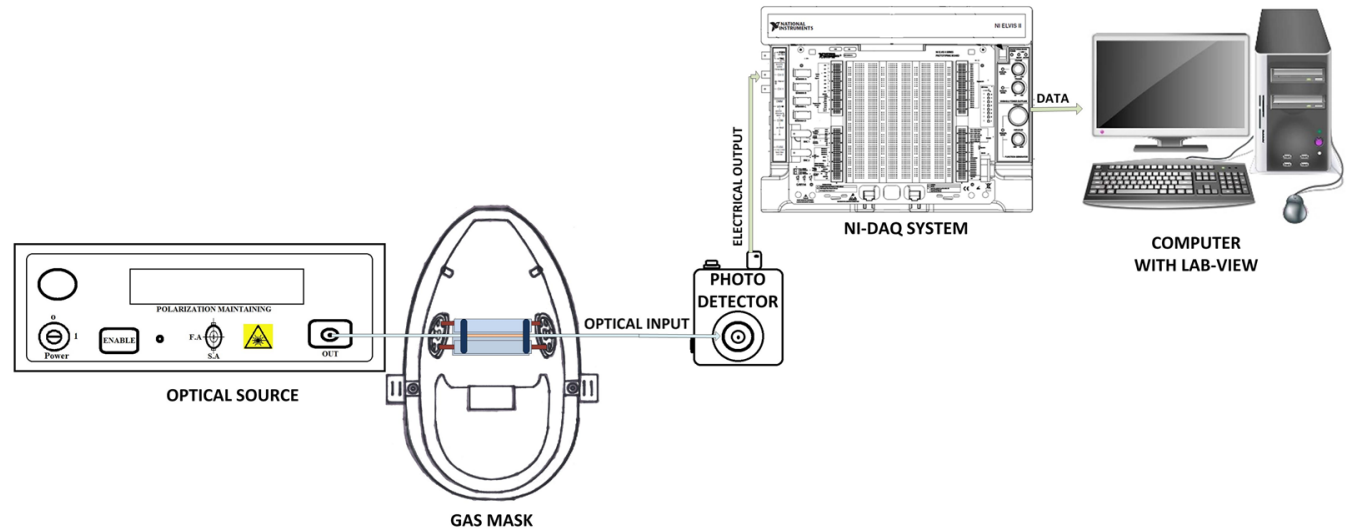

Figure 2. Schematic diagram of the breath monitoring experimental setup.

textile-based respiratory sensing system by integrating polymeric optical fibers (POFs) into a carrier fabric to form a wearable sensing system. This respiratory sensing system was placed on a human torso and operated based on the measurement of light intensity changes in POFs as a result of applied pressure. Chen et al [5] proposed a tapered-tip fiberoptic fluorescence oxygen sensor for monitoring human breathing. A POF based force change based breathing rate sensor was reported by Mohanty et al [6]. Kang et al [7] designed an optical fiber-based breathing sensor by applying a multi-layered inorganic nanocluster and polymer thin films on the distal ends of optical fibers. The sensor detects the variation in relative humidity that occurs between inhaled and exhaled breath and features non-invasive, fast and reliable monitoring of respiratory airflow. Previously, we also reported a humidity based breathing sensor with an agarose infiltrated photonic crystal fiber interferometer [8]. Smith et al [9] proposed a non-invasive temperature based respiration sensor to track the breathing cycle during respiratory gating. Respiration sensors utilizing optical fiber bending effects have been regarded as a promising technique and widely investigated [10-13], for example. Witt et al [10] developed a monitoring system for continuous measurement of abdominal and thoracic respiratory movement using optical fiber sensors based on macrobending effects. All the above mentioned sensors either suffer from coating fabrication difficulties (time consuming, non-uniformity, limited life time and poor stability) or from a complexity of the sensing system. For example, a fiber Bragg grating (FBG)

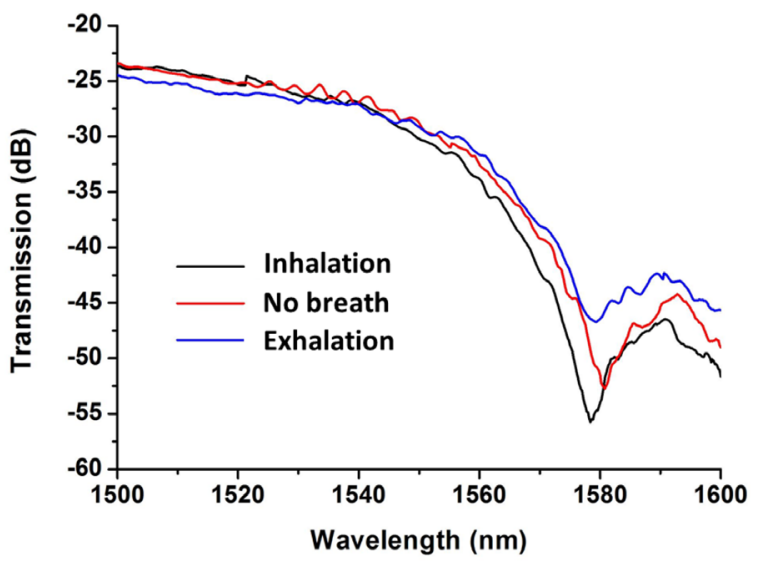

Figure 3. Spectral responses of the SMS breath sensor during inhalation and exhalation process.

based breath sensor normally requires monitoring of the Bragg wavelength shift [14], so that the system suffers from a relatively high cost for both interrogation equipment (for example, a wavelength interrogation system) and the FBG sensor itself.

Single mode-multimode-single mode (SMS) fiber structures have been intensively investigated as refractive index (RI), temperature and humidity sensors over the past decade [15-19]. In our previous report, we have proved that an SMS fiber structure is sensitive to macro bending [20,21]. In this letter, for the first time we propose a simple, cost effective breathing sensor based on an SMS fiber structure, which 


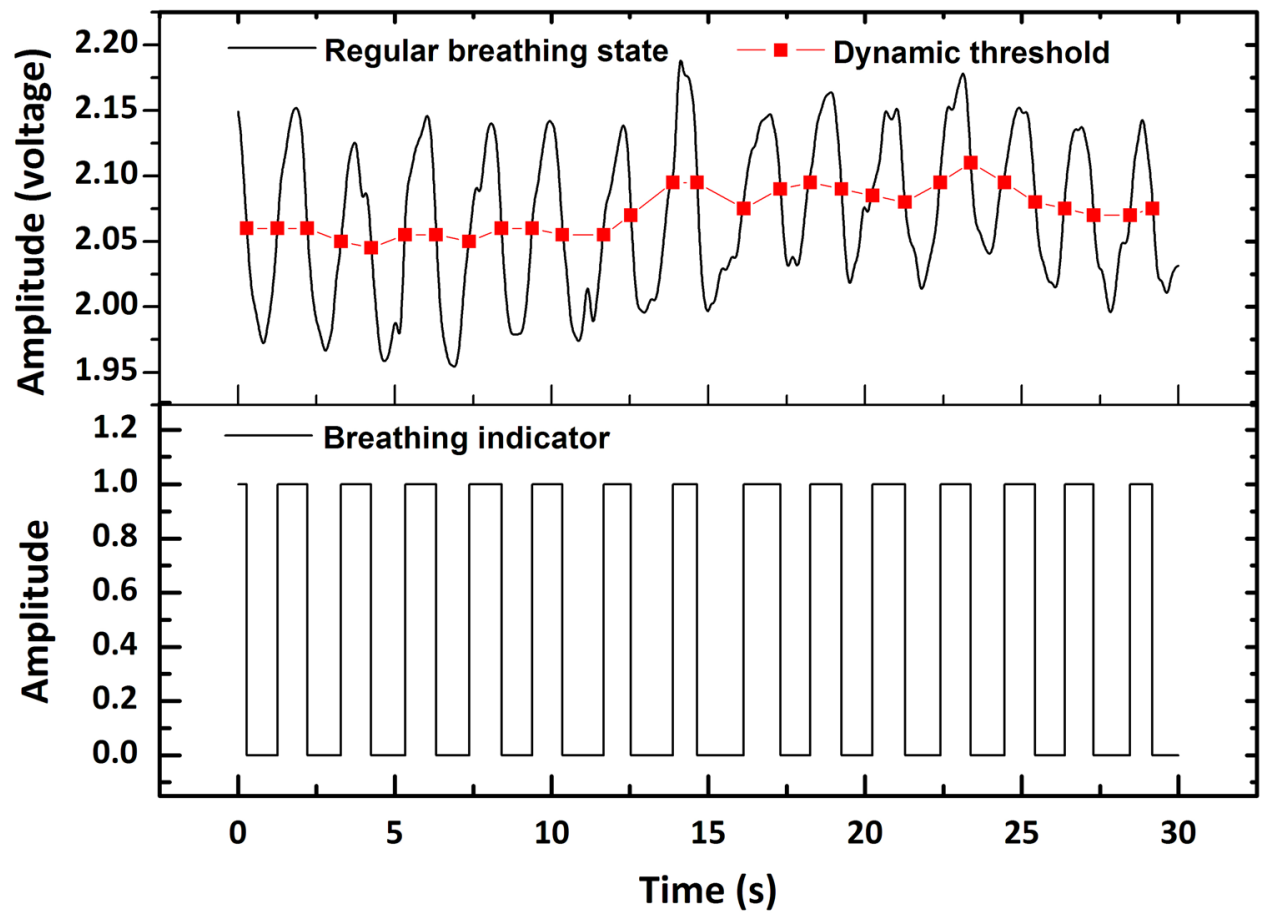

Figure 4. Experimentally recorded breathing pattern and dynamic threshold in real time (upper plot) and the corresponding breath analysis program response (lower plot) to a regular breathing pattern.

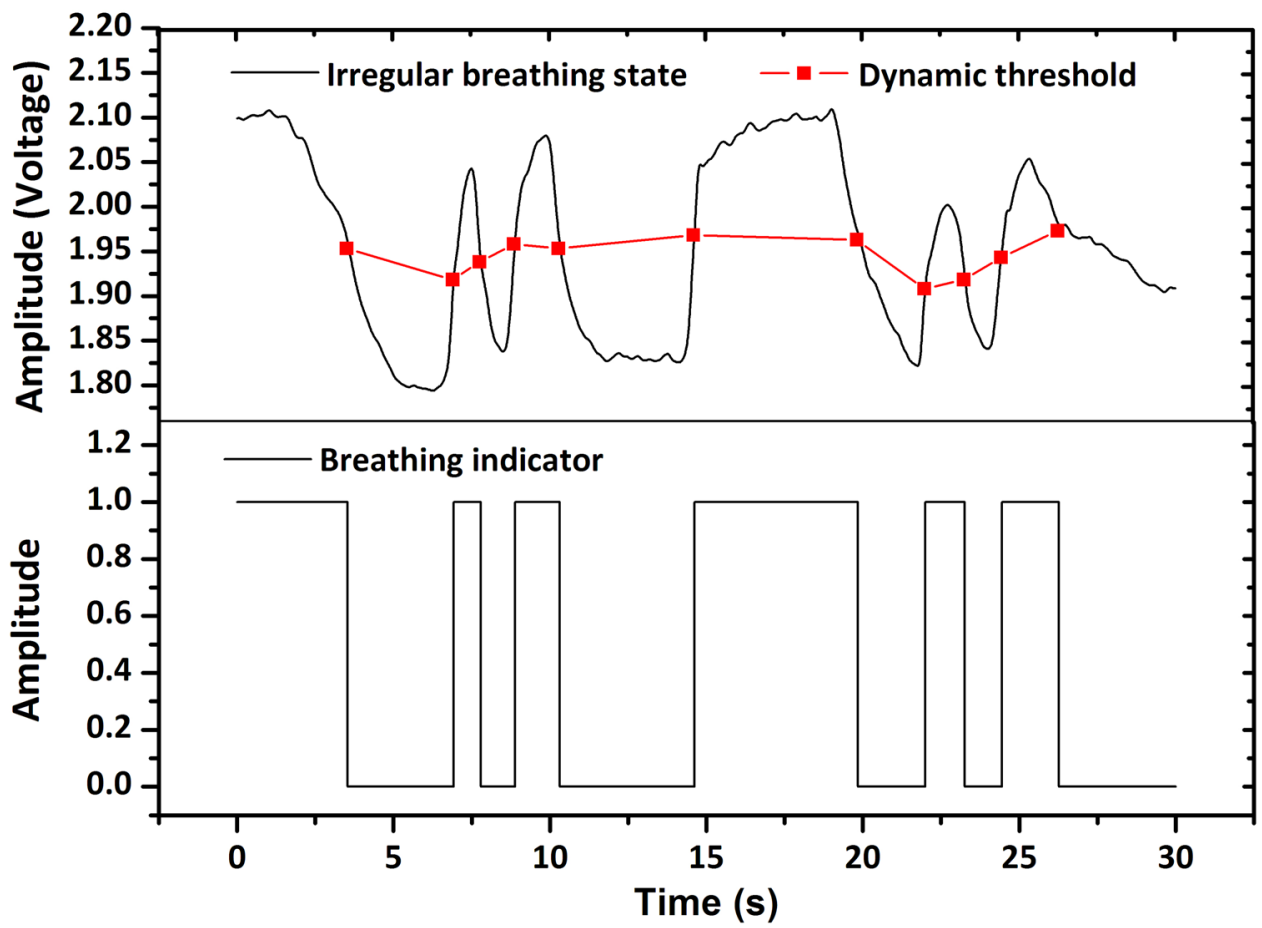

Figure 5. Experimentally recorded breathing pattern and dynamic threshold in real time (upper plot) and the corresponding breath analysis program response (lower plot) to an irregular breathing pattern.

monitors the power variations (instead of wavelength shift for an FBG sensor), showing a fast and reliable response to different types of breathing conditions (regular, irregular, slow and urgent breath state). The proposed breath sensor does not require any coating (and hence has long life-time) and is mechanically robust.

\section{Principle and experimental setup}

Figure 1(a) shows a schematic diagram of the proposed SMS fiber structure. Light entering from a single mode fiber (SMF) to the multimode fiber (MMF, typically has a step-index profile), excites multiple modes propagating along the MMF 


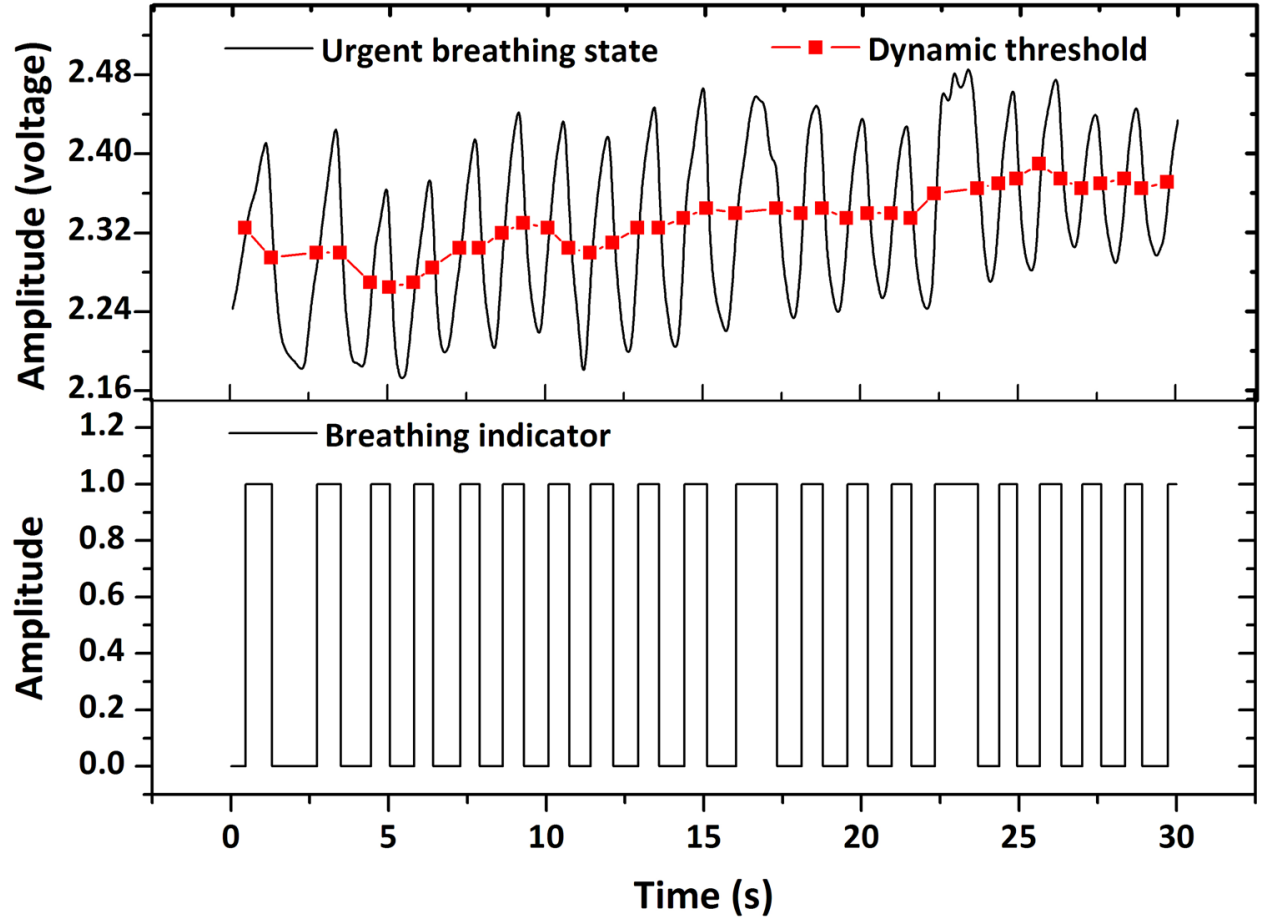

Figure 6. Experimentally recorded breathing pattern and dynamic threshold in real time (upper plot) and the corresponding breath analysis program response (lower plot) to an urgent breathing pattern.

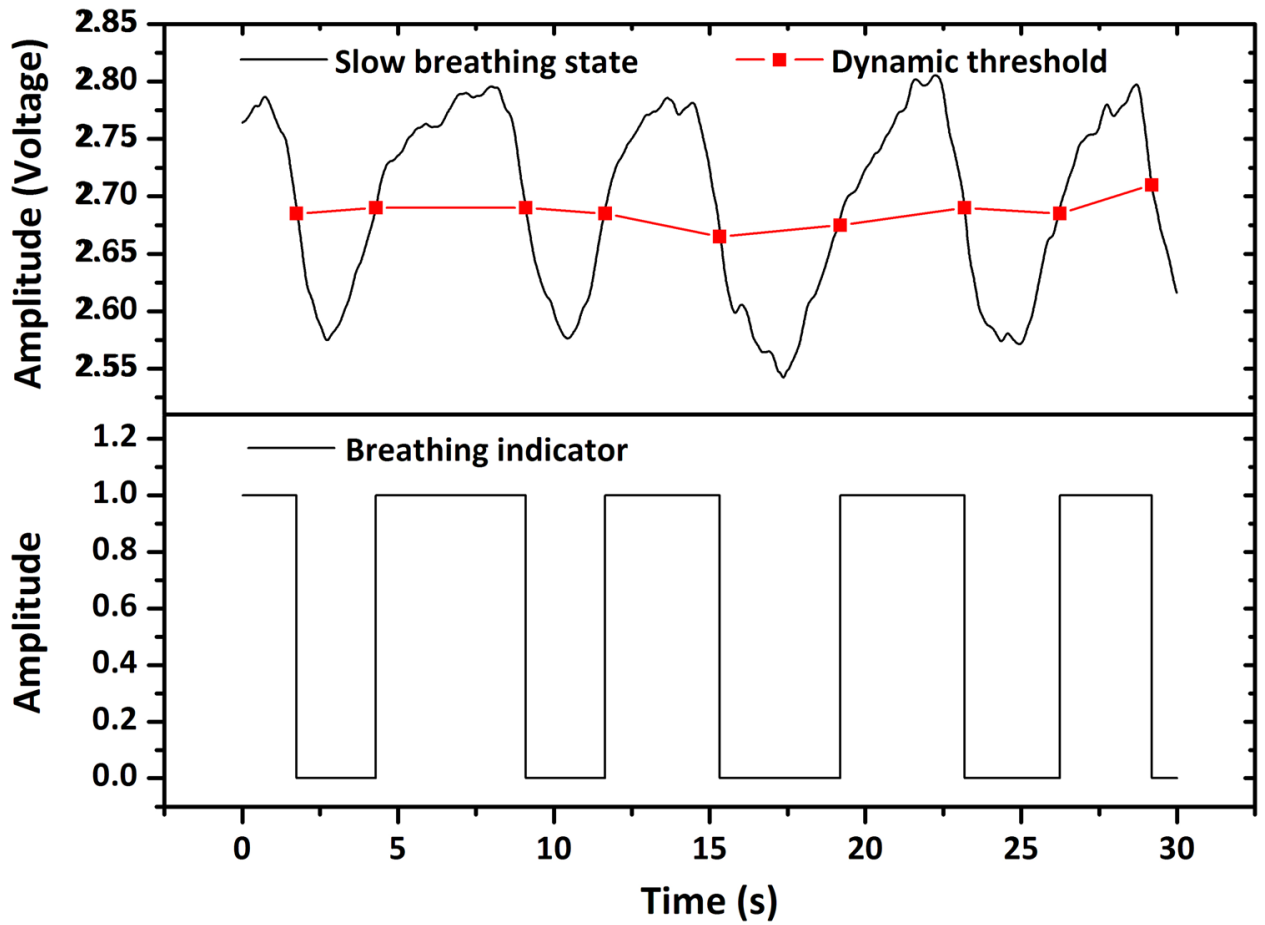

Figure 7. Experimentally recorded breathing pattern and dynamic threshold in real time (upper plot) and the corresponding breath analysis program response (lower plot) to a slow breathing pattern.

section. The field in the MMF section will be distributed symmetrically with the respect to the fiber axis due to the symmetrical RI profile distribution. However, if the MMF is bent, the RI distribution is no longer symmetric so that even a minor bend within the MMF section will introduce significant power variations at the output of the SMS fiber structure [22]. If such an SMS sensor is embedded within a flexible membrane positioned near the patient's mouth, the breathing process will introduce macro bends to the SMS structure and hence will result in light power variations at the output of the SMS fiber structure. By monitoring the light power variation rate, the breathing rate can be measured. In our experiments, a short section of a MMF (AFS105/125Y with a core diameter of $105 \mu \mathrm{m}$ and cladding diameter of $125 \mu \mathrm{m}$ ) with a length 
of circa $20 \mathrm{~mm}$ was fusion spliced between two singlemode (SMF-28) fibers and attached to a thin plastic film within an oxygen mask as shown in figure 1(b). The SMS fiber was fixed on a plastic film which was wrapped around two thin support rods. The distance between the SMS based breathing sensor and patient's nose was set to approximately $50 \mathrm{~mm}$ and can be adjust in real applications depending on the condition of the patient. When the patient is breathing, the air flowing from their nose/mouth will induce pressure on the thin plastic film and hence will bend the SMS sensor, resulting in power variations at the output of the sensor.

Figure 2 illustrates the schematic diagram of the experimental setup used for breath state monitoring. The broadband optical source (Thor Labs S5FC 1550P) used in the experiment is a superluminescent diode with a central wavelength of $1550 \mathrm{~nm}$. Light transmitted through the SMS structure is converted into an electrical signal by a photo-detector and acquired with a data acquisition system (NI-ELVIS II), and then analysed using a program based on the Lab-View software.

\section{Results and discussion}

Figure 3 illustrates the transmission spectral response during the inhalation and exhalation process. The inhalation and exhalation cycles will result in different forces applied on the plastic film which in turn will induce different bend radii in the SMS fiber structure and hence different spectral responses are recorded by the Photodetector.

Figure 4 illustrates the breath pattern for a regular breath state tested during $30 \mathrm{~s}$. The upper plot (black line) shows the votage change (representing the transmitted power variations detected by the photodetector) corresponding to the real time breathing response. During the exhale cycle, the air flow from the patient's nose will introduce bent to the SMS sensor, resulting in a power variation transmitted to the photodector, therefore the valleys and peaks of the photodetector response in figure 4 represent inhalation and exhalation cycles respectively. It is noted that due to the fact that the SMS sensor itself is bound to a thin plastic film it is difficult to ensure it is attached in a perfectly straight position. In addition, the power detected by the photodetector doesn't remain constant due to inevitable environmental fluctuations such as air flow, vibration, incomplete recovery of the plastic film shape, etc. It is hence impossible to set a fixed value as the threshold for determining either inhalation or exhalation states. However, this problem can be solved by setting a dynamic threshold as an average value of all the adjacent peak and valley pairs as shown in figure 4 with a red spot line plot. The lower plot in the figure 4 is the breathing cycle indicator calculated from the breathing pattern in the upper plot using a dynamic threshold. In the experiment, we define the value lower than the threshold as breathing state ' 0 ' which corresponds to an inhalation, while the value higher than the threshold-as breathing state ' 1 ' which corresponds to an exhalation. It is clear from the figure 4 that the SMS fiber structure based breathing sensor can be easily used for monitoring the regular breathing process.
In order to demonstrate that our breathing sensor system is capable of monitoring various breathing states in real time, three more breathing behaviors were tested and the corresponding breathing patterns were shown in figures 5-7 with respect to irregular, urgent and slow breathing. One can see that our sensor can easily distinguish between the above different types of breathing conditions.

\section{Conclusion}

A simple SMS fiber structure based breathing sensor has been proposed. The sensor can be used in strong electric/magnetic field and radioactive environments such as during MRI and CT examinations where traditional electronic sensors could not be applied. By detecting the transmitted optical power variations due to fiber bending resulting from inhalation and exhalation cycles, people's breathing state can be monitored in real time. The proposed sensor can help to easily distinguish between the different types of breathing conditions from a regular and irregular breathing to an urgent and slow breathing patterns. It features advantages of easy fabrication, low cost, fast response and potentially good resistance to environmental contamination.

\section{Acknowledgment}

This work is supported by DIT FIOSRAIGH 2012 (Dean of Graduate Studies Award); Open Fund of IPOC (BUPT).

\section{References}

[1] Braun S R 1990 Clinical Methods, the History, Physical, and Laboratory Examinations (Stoneham, MA: Butterworth Publishers) ch 43

[2] Al-Khalidi F Q, Saatchi R, Burke D, Elphick H and Tan S 2011 Respiration rate monitoring methods: a review Pediatr. Pulmonol. 46 523-9

[3] Dempsey M F and Condon B 2001 Thermal injuries associated with MRI Clin. Radiol. 56 457-65

[4] Krehel M, Schmid M, Rossi R M, Boesel L F, Bona G-L and Scherer L J 2014 An Optical fibre-based sensor for respiratory monitoring Sensors 14 13088-101

[5] Chen R, Formenti F, Obeid A, Hahn C E W and Farmery A D 2013 A fibre-optic oxygen sensor for monitoring human breathing Physiol. Meas. 34 N71-81

[6] Mohanty L and Kuang K S C 2010 A breathing rate sensor with plastic optical fiber Appl. Phys. Lett. 97073703

[7] Kang Y, Ruan H, Wang Y, Arregui F J, Matias I R and Claus R O 2006 Nanostructured optical fibre sensors for breathing airflow monitoring Meas. Sci. Technol. 17 1207-10

[8] Mathew J, Semenova Y and Farrell G 2012 A miniature optical breathing sensor Biomed. Opt. Exp. 3 3325-31

[9] Smith A, Kim S, Serago C, Hintenlang K, Pooley R and Hintenlang D 2015 Development of an MR and CT compatible non-invasive temperature based optical fiber respiration sensor for use in radiotherapy World Congress on Medical Physics and Biomedical Engineering (Toronto, Canada) pp 603-6

[10] Witt J et al 2012 Medical textiles with embedded fiber optic sensors for monitoring of respiratory movement IEEE Sens. J. 12 246-54 
[11] Yang X, Chen Z, Elvin C S M, Janice L H Y, Ng S H, Teo J T and Wu R 2015 Textile fiber optic microbend sensor used for heartbeat and respiration monitoring IEEE Sens. J. 15 757-61

[12] Hu H F, Sun S J, Lv R Q and Zhao Y 2016 Design and experiment of an optical fiber micro bend sensor for respiration monitoring Sensors Actuators A 251 126-33

[13] Di H, Sun S and Che Y 2016 Respiration measurement using fibre-optic deformation sensor J. Mod. Opt. 1-7

[14] Dziuda L, Skibniewski F W, Krej M and Lewandowski J 2012 Monitoring respiration and cardiac activity using fiber Bragg grating based sensor IEEE Trans. Bio-med. Eng. 59 1-9

[15] Wu Q, Semenova Y, Wang P and Farrell G 2011 High sensitivity SMS fiber structure based refractometeranalysis and experiment Opt. Exp. 19 7937-44

[16] Sun G, Wu G, Wang F, Tang R and Qiu G 2016 High-stress resistance fiber refractometer based on MMF sandwiched between two SMF half-tapers IEEE Photonic Tech. Lett. 28 1336-9

[17] An J, Jin Y, Sun M and Dong X 2014 Relative humidity sensor based on SMS fiber structure with two waist-enlarged tapers IEEE Sens. J. 14 2683-6
[18] Tripathi S M, Kumar A, Varshney R K, Kumar Y B P, Marin E and Meunier J P 2009 Strain and temperature sensing characteristics of single-mode-multimode-single-mode structures J. Lightwave Technol. 27 2348-56

[19] Del Villar I, Socorro A B, Corres J M, Arregui F J and Matias I R 2013 Optimization of sensors based on multimode interference in single-mode-multimodesingle-mode structure J. Lightwave Technol. 31 3460-8

[20] Wu Q, Semenova Y, Wang P, Hatta A M and Farrell G 2011 Experimental demonstration of a simple displacement sensor based on a bent single-mode-multimodesingle-mode fiber structure Meas. Sci. Technol. 22025203

[21] Wu Q, Yang M, Yuan J, Chan H P, Ma Y, Semenova Y, Wang P, Yu C and Farrell G 2014 The use of a bend singlemodemultimode-singlemode (SMS) fiber structure for vibration sensing Opt. Laser Technol. 63 29-33

[22] Schermer R T and Cole J H 2007 Improved bend loss formula verified for optical fiber by simulation and experiment J. Quantum Electron. 43 899-909 